\title{
REPORT ON THE CAPE PARTICIPATION IN THE SOUTHERN REFERENCE STAR PROJECT
}

\author{
R. H. TUCKER \\ Royal Greenwich Observatory, Herstmonceux, England
}

The Gill Transit Circle at the Cape Observatory has observed zones $-40^{\circ}-52^{\circ}$ and $-30^{\circ}-40^{\circ}$ of SRS and is now observing zone $-52^{\circ}-64^{\circ}$, which is $80 \%$ complete. In addition to fundamental stars selected to define the FK4 system in the zone of observation, clock stars and azimuth stars are also observed so that the observations can be subsequently referred to a more independent or fundamental system.

Observations of the Bright Star list associated with SRS have also been made contemporaneously throughout the period (1961-1973).

The current reductions are being performed at Herstmonceux. Data forms completed and checked by hand at the Cape are sent to Herstmonceux, where they are encoded on magnetic tape by means of a Matador keyboard machine. Errors in data are corrected after consultation by correspondence, and the ledgered results are analysed and discussed in the Cape Meridian Department.

Preliminary investigation of the observations of FK4 stars for 1966-1969 shows that the instrumental declination system is practically free from clamp differences in $\Delta \delta_{\delta}$, and has no serious $\Delta \delta_{\alpha}$ term. The R.A. observations are likewise free from clamp effect, but show some unexpected anomalies in the dependence of SD on ZD.

Gill's transit-circle is now near the end of a very active career that began in 1903. It would not be practicable to remove this telescope to the Sutherland observing station of SAAO, now established in the Karroo, and it seems far more fitting that it should remain on its own site as an example of instrumental design of the beginning of the twentieth century that was several decades ahead of its competitors.

To take its place as part of the SAAO's astrometric effort, we hope to develop a transportable automatic transit circle that can be used at Herstmonceux and Sutherland to give fundamental positional coverage in both hemispheres.

\section{DISCUSSION}

Edmondson: As a non-expert I am shocked at the concept of a 'transportable' transit circle. Could you explain in more detail this departure from the traditional concept of leaving the instrument undisturbed for long periods of time?

Tucker: We are perhaps nowadays rather more confident in our ability to attain and control adequate instrumental rigidity. It is mainly a question of taking advantage of modern engineering techniques and materials.

Klock: In Washington we feel that the success of the stability of the 6-in. T.C. may be attributed to 2 important factors:

(1) The design and stability of the pier foundation for the instrument, and

(2) The stability of the azimuth marks. 